\title{
CHARACTERISTICS OF RESILIENCE IN HONG KONG OLDER ADULTS: A QUALITATIVE STUDY
}

\author{
Pak Kwong Chung ${ }^{13^{*}}$, Chun $\mathrm{Hu}^{2,4}$, \& Chun-Qing Zhang ${ }^{2,5}$ \\ ${ }^{1} D P E$. \\ ${ }^{2} P h . D$ \\ ${ }^{3}$ Department of Sport, Physical Education and Health, Hong Kong Baptist University, Hong Kon (China) \\ ${ }^{4}$ Student Mental Health Education Center, Northwestern Polytechnical University, Xi'an (China) \\ ${ }^{5}$ Department of Psychology, Sun Yat-sen University, Guangzhou (China)
}

\begin{abstract}
Introduction: Resilience, which is defined as the process of adapting well in the face of adversity, trauma, tragedy, threats or significant sources of stress, is important for older adults to maintain a healthy life. This qualitative study aimed to identify the characteristics that contribute to resilience among a group of community-dwelling older adults in Hong Kong.

Methods: Individual and focus group interviews were conducted to collect information on life adverse events, attitudes towards adversity, and beliefs underlying the approaches to overcoming adversity among 25 Chinese older adults $(2 \mathrm{M}$ and $23 \mathrm{~F})$ aged 69 to 100 years old $(\mathrm{M}=80.00, \mathrm{SD}=39.08)$. The transcripts were analyzed using qualitative content analysis.

Results: Seven characteristics were emerged under the three factors, including equanimity, positive attitudes towards life, meaningfulness, and self-reliance (internal factor), social support and environmental support (external factor), and spirituality and faith (existential factor).

Conclusion: In addition to identifying the seven characteristics that contribute to resilience, this study also identified "taking part in physical activity" as an individual resource contributing to resilience. The study also found "government support" is an important environmental factor contributing to positive adaptation to stressful life of the elderly in Hong Kong. The results and findings may facilitate the development of interventions on enhancing older adults' resilience.
\end{abstract}

Keywords: Resilience, adversity, older adults, qualitative study.

\section{Introduction}

When older adults face adversity, it is sometimes difficult for them to bounce back (Hildon, Smith, Netuveli, \& Blane, 2008). Under these circumstances, resilience is a particularly important resource for the elderly people to encounter adversity. There is no uniform definition of resilience. It was initially considered as an ability to bounce back or cope successfully despite substantial adversity (Rutter, 1985). Other researchers have defined resilience as the outcome of successful adaptation in spite of threatening or challenging environment (Masten, Best, \& Garmezy, 1990), as well as the process of adapting well in the face of adversity, trauma, tragedy, threats or significant sources of stress (Tugade \& Fredrickson, 2004). Although there is substantial evidence that resilience is a synthesis of a variety of resources, the knowledge of resilience in older adults in a Chinese cultural context such as Hong Kong is insufficient (Cheung \& Kam, 2012). This qualitative study aimed to identify the characteristics that contribute to resilience among a group of community-dwelling older adults in Hong Kong.

\section{Methods}

Convenience sampling was used to recruit older adults from the community senior centres in Hong Kong, with the following criteria: (a) Hong Kong Chinese older adults who were at least 65 years old; (b) able to speak Cantonese; and (c) had good visual or auditory acuity or fundamental literacy. A total of 25 older adults ( $2 \mathrm{M}$ and $23 \mathrm{~F}$ ) aged 69 to 100 years old $(\mathrm{M}=80.00, \mathrm{SD}=39.08)$. The participants were recruited according to the principle of voluntariness and their data were kept confidential. Informed 
consent letters were signed before the interviews. The semi-structured individual interviews and focus groups were subsequently conducted in the centres. The interview recordings were transcribed verbatim and the transcripts of the interviews were analyzed using qualitative content analysis. The analytic induction to describe the perceptions of participants' perspectives (Elo \& Kyngäs, 2008) was adopted. Data was analyzed independently by two coders with psychology background and expertise in resilience. Consensus were reached after discussion between the two coders. Remained discrepancies were resolved by consulting a third coder who is a psychology professor with expertise in resilience.

\section{Results}

As reflected by the interviewees, resilience was a dynamic process that occurs over time and that involved different factors of resilience that could be internal, external or existential ones. Accordingly, seven themes were identified, including equanimity, positive attitudes towards life, meaningfulness, self-reliance, social support, environmental support, spirituality and faith.

\section{Discussion}

The current qualitative study has developed systematic descriptions of sources of resilience from the perspective of older adults in Hong Kong. For example, 'contentment is happiness' and 'live in the moment' could be considered specific instances of the source described in the literature as 'focus on the present' (Lopez-Fuentes \& Calvete, 2015). In addition, it is found that spirituality and faith are an existential source of resilience, which has been discussed in the literature as a helpful factor in coping with diseases (Pentz, 2005). Another contributor to resilience identified was 'taking part in physical activity', which has also been found in a previous study (Takahashi et al., 2016), although it has sometimes been classified as an individual resource (Lopez-Fuentes \& Calvete, 2015). Lastly, it should be emphasized that the current study is the first that identifies government support as an important factor in environmental support, which contributes to positive adaptation to the stressful life of the elderly in Hong Kong.

\section{Conclusion}

The current study identifies seven characteristics that contribute to resilience, including equanimity, positive attitudes towards life, meaningfulness, self-reliance, social support, environmental support, spirituality and faith. The findings may facilitate the development of interventions on enhancing older adults' resilience in Hong Kong or other places.

\section{References}

Cheung, C., \& Kam, P. K. (2012). Resiliency in older Hong Kong Chinese: Using the grounded theory approach to reveal social and spiritual conditions. Journal of Aging Studies, 26(3), 355-367.

Elo, S., \& Kyngäs, H. (2008). The qualitative content analysis process. Journal of Advanced Nursing, 62(1), 107-115.

Hildon, Z., Smith, G., Netuveli, G., \& Blane, D. (2008). Understanding adversity and resilience at older ages. Sociology of Health \& Illness, 30(5), 726-740.

Lopez-Fuentes, I., \& Calvete, E. (2015). Building resilience: A qualitative study of Spanish women who have suffered intimate partner violence. American Journal of Orthopsychiatry, 85(4), 339-351.

Masten, A. S., Best, K. M., \& Garmezy, N. (1990). Resilience and development: Contributions from the study of children who overcome adversity. Development and Psychopathology, 2, 425-444.

Pentz, M. (2005). Resilience among older adults with cancer and the importance of social support and spirituality-faith. Journal of Gerontological Social Work, 44(3-4), 3-22.

Rutter, M. (1985). Resilience in the face of diversity: protective factors and resistance to psychiatric disturbance. British Journal of Psychiatry, 147, 598-611.

Takahashi, K., Sase, E., Kato, A., Igari, T., Kikuchi, K., \& Jimba, M. (2016). Psychological resilience and active social participation among older adults with incontinence: A qualitative study. Aging \& Mental Health, 20(11), 1167-1173.

Tugade, M. M., \& Fredrickson, B. L. (2004). Resilient individuals use positive emotions to bounce back from negative emotional experiences. Journal of Personality and Social Psychology, 86(2), 320-333. 